\title{
ESTACIONALIDAD TURÍSTICA EN LAS PROVINCIAS ESPAÑOLAS: MEDICIÓN Y ANÁLISIS
}

\author{
Juan Antonio Duro \\ Francesc Xavier Farré \\ Universitat Rovira i Virgili. Tarragona
}

\section{RESUMEN}

En este trabajo se lleva a cabo un análisis de la concentración temporal de la demanda turística en las provincias españolas para el período 1999-2012. El análisis se realiza a partir del uso de diferentes índices sumario, que recogen diferentes sensibilidades de las medidas en relación a la distribución temporal de la actividad, y de los datos de pernoctaciones hoteleras ofrecidos por el INE. El trabajo realiza un análisis descriptivo de la concentración provincial, de su evolución temporal y, finalmente aborda el grado de correlación entre la concentración y diferentes vectores explicativos potenciales.

Palabras clave: Estacionalidad; provincias españolas; medición.

\section{Tourist season in the spanish provinces: measurement and analysis}

\section{ABSTRACT}

In this paper we carried out an analysis of the temporal concentration of tourism demand in the Spanish provinces for the period 1999-2012. The analysis is carried out through the use of different summary indices, which reflect different sensitivities of the measures in relation to the temporal distribution of the activity, and overnights data provided by INE. The analysis performed a descriptive analysis of provincial concentration, their temporal evolution and finally addresses the correlation between concentration and different potential explanatory variables.

Keywords: Seasonality; Spanish provinces; measurement.

Recibido: 21 de diciembre de 2013

Devuelto para su revisión: 28 de abril de 214

Aceptado: 26 de marzo de 2015

Departamento de Economía. Universitat Rovira i Virgili. Avinguda Universitat, 1. 43204 Reus. TARRAGONA (España).E-mail: juanantonio.duro@urv.cat 


\section{INTRODUCCIÓN}

La estacionalidad constituye uno de los principales desequilibrios de los destinos y regiones turísticas. Sus nocivas consecuencias emergen de manera más pronunciada en los destinos desarrollados, plasmándose en términos laborales, medioambientales y de eficiencia económica. Implica, de hecho, uno de los parámetros esenciales para promover un desarrollo sostenible de la actividad turística en el territorio y típicamente es objeto, por ejemplo, de un lugar destacado en la planificación estratégica turística territorial.

Una de las definiciones más aceptadas de lo que se entendería por estacionalidad se la debemos a Butler (2001, p. 5), conocido autor que apunta al concepto como la aparición de un desequilibrio temporal en la actividad turística de los destinos, de demanda o de oferta. En este sentido, y por consistencia con dicha noción de «desequilibrio», el fenómeno de la estacionalidad debería medirse cuantitativamente a través de índices de desequilibrio, o de desigualdad, como comúnmente se refiere. De hecho, antes que estacionalidad cabría hablar, con mayor precisión, de concentración o desigualdad temporal de la actividad turística.

Dada la importancia atribuida, es lógico que haya aparecido la conveniencia de investigar, desde un enfoque científico, diferentes aspectos asociados al conocimiento de este fenómeno. En este sentido, Koenig-Lewis y Bischoff (2005), por ejemplo, han estructurado de una forma muy útil las principales necesidades científicas a este respecto. Sintéticamente, estos autores establecen seis grandes áreas de investigación, con importantes lagunas existentes en casi todas ellas: la definición de estacionalidad, las causas, los impactos, las implicaciones de políticas, el conocimiento del turista y los aspectos de medición. En este sentido, este trabajo se focaliza, en esencia, en el último de los aspectos.

En particular, sorprende un tanto la poca atención que la literatura ha dedicado precisamente a este último aspecto, el de la medición de la estacionalidad y sus implicaciones en función de las medidas y posibilidades (Koenig-Lewis y Bischoff, 2005; López y López, 2005; Martín et al., 2014). En el caso español, en todo caso, contamos por ejemplo con los trabajos de Fernandez-Morales (2003), López y López (2006), Capó et al. (2007), Duro (2008), Fernandez-Morales y Cruz-Mayorga (2008) o Martín et al. (2014), los cuáles básicamente utilizan el coeficiente de Gni para medir la estacionalidad para diferentes destinos, desde las Islas Baleares hasta la Costa del Sol o las CCAA ${ }^{1}$.

Fundamentalmente, el punto de interés es que las medidas utilizadas pueden dar resultados distintos en función de los datos en cada caso. En particular, en este trabajo se revisan las propiedades de algunas de las principales medidas de desigualdad temporal que se pueden utilizar, destacando, entre otros aspectos, su diferente sensibilidad ante los cambios en la distribución temporal. En segundo lugar, el trabajo realiza una aplicación empírica para las provincias españolas en el período 1999-2012. Los datos proxy de la actividad turística utilizados provienen de las series de pernoctaciones hoteleras del INE. Como es bien sabido, estas pernoctaciones son sólo una parte de la demanda. En todo caso, implican la mayor parte de las pernoctaciones regladas año a año en todas las provincias, a falta de datos rigurosos y suficientemente completos sobre la demanda en el formato de

1 En el ámbito internacional una aplicación reciente es la llevada a cabo por Cuccia y Rizzo (2011). 
alojamiento residencial ${ }^{2}$. El enfoque del trabajo es general y, por tanto, no va a explorar los patrones y situaciones de las diferentes provincias con detenimiento. Adicionalmente, se repasa tentativamente la significatividad de algunas variables en relación a la estacionalidad comparada a través de diversos análisis correlativos preliminares.

El paper, pues, se estructura de la forma siguiente. En la sección segunda se repasan los principales aspectos metodológicos relativos a la medición de la estacionalidad turística, cuando se concibe ésta en términos del desequilibrio existente cada año en la distribución temporal de la actividad. En la sección tercera se realiza un cálculo de la concentración temporal de la actividad turística en todas las provincias españolas a lo largo del período 1999-2012. En la sección cuarta se sintetizan algunos análisis correlativos con el objeto de establecer una evaluación preliminar sobre factores que pueden estar asociados a los niveles y las diferencias en la concentración temporal. Finalmente, una sección acoge las principales conclusiones obtenidas del trabajo.

\section{ASPECTOS METODOLÓGICOS ACERCA DE LA MEDICIÓN DE LA ESTA- CIONALIDAD}

Baron (1975) fue uno de los primeros autores en subrayar la estacionalidad turística como uno de los principales desequilibrios de la actividad turística. Han surgido en la literatura diferentes definiciones de estacionalidad. A pesar de la diversidad, bien es verdad que una amplia mayoría de autores hace referencia a la aparición en el destino de una regularidad sistemática dentro del año en los flujos. El propio BarOn (1975), por ejemplo, habla de ésta como los efectos que ocurren cada año con mayor o menor intensidad en el destino. Allock (1994) la define concretamente en términos de la concentración de los flujos de turistas en cortos períodos de tiempo del año. En todo caso, la definición que más nos satisface es la de Butler (2001, p5), que es más abierta que las anteriores: «sería el desequilibrio temporal en el fenómeno del turismo, que puede ser expresado en términos de dimensiones de tales elementos como el número de visitantes, el gasto de los visitantes, el tránsito en autopistas y otras formas transporte, empleo y entradas de atracciones».

Tomando, pues, la definición de Butler como referencia, la estacionalidad debería medirse a través de indicadores de desequilibrio temporal intra-año o, si se quiere, de desigualdad. En este sentido, una vía inmediata consistiría en extraer algunos de los aprendizajes surgidos de la literatura sobre medición de la desigualdad. Autores como Chakravarty (1990) o Cowell (1995) han ofrecido interesantes revisiones al respecto. En esencia, esta literatura ha derivado ciertos axiomas básicos que toda medida debe satisfacer y ha analizado las características asociadas al uso de diferentes medidas. Entre ellas, algunas de las más conocidas son el coeficiente de Gini, los índices de la familia de Theil o el Coeficiente de Variación. El punto esencial aquí es que la aplicación de cada una de ellas puede generar resultados distintos en función de cómo pondera cada una las distancias de las diferentes observaciones. Vamos a revisar en esta sección las características peculiares de la lista de medidas anterior.

2 Por ejemplo, según el Instituto de Estudios Turísticos, el 65\% de los turistas internacionales se alojan en establecimientos hoteleros, mientras que este porcentaje se reduce al $18 \%$ en el caso de los turistas españoles. 
Ante todo, se ha de puntualizar que, en el caso que nos ocupa, se está examinando la distribución temporal de la actividad turística a lo largo del año en un territorio y/o destino en cuestión. En este sentido, las observaciones temporales, que son las unidades básicas para el análisis, pueden ser variadas. Una opción razonable sería considerar los meses del año como unidades básicas, lo cual daría al análisis un grado de detalle interesante, con los datos disponibles, y coincidiría con la unidad de seguimiento temporal típico de las empresas turísticas y las administraciones. A este respecto, sabemos que no es posible, de manera homogénea en España, detallar aún más la actividad turística. En todo caso, podría elegirse una base temporal más agregada para el análisis, por ejemplo trimestres, cuatrimestres o temporadas. En este sentido, se perdería precisión analítica pero, por ejemplo, se podrían evitar las consecuencias del efecto calendario en algunos de los ejercicios, por ejemplo, en lo que respecta a la ubicación de la semana santa. El punto clave sería, entonces, establecer los límites de estos subperíodos, lo cual no es trivial.

Por tanto, supongamos, por ejemplo, que la concentración temporal de la actividad va a tomar el mes como unidad de referencia. Entonces, las medidas de desigualdad en realidad serían medidas de desigualdad intermensual de la actividad. Probablemente la medida más conocida destinada a medir de manera sintética el grado de desigualdad (dispersión) sea el coficiente de Gini (Gini, 1912), que se relaciona de manera gráfica con la conocida curva de Lorenz. Una de las fórmulas más convencionales de esta medida es la siguiente (ya adaptada al análisis de la concentración temporal turística):

$$
G=\frac{1}{2 \mu} \sum_{i} \sum_{j} p_{i} p_{j}\left|y_{i}-y_{j}\right|
$$

donde $\mathrm{p}_{\mathrm{i}} \mathrm{i} \mathrm{p}_{\mathrm{j}}$ son los pesos de las observaciones (meses o temporadas); $\mathrm{y}_{\mathrm{i}}$ es, en nuestro caso, la demanda hotelera mensualy $\mu$ es la media anual.

Nótese que i, j pueden ser dos meses cualesquiera del año. Asimismo, nótese que las ponderaciones de cada observación, en su aplicación al análisis de estacionalidad, son iguales para todos los períodos comparados y equivalentes a $1 / \mathrm{n}$, donde «n» es el número de periodos considerados. Así las cosas, si el análisis se centra en una comparación mensual «n» es igual a 12 y si, por ejemplo, es cuatrimestral seria $1 / 3$.

En particular, se han ensalzado tres características respecto a esta medida (Wanhill, 1980; o Lundtorp, 2001). Primero, su reducida dependencia en relación a los cambios en los meses peak; segundo, su elevada estabilidad comparada con otras medidas y tercero, su reducida sensibilidad a los valores (meses) extremos. Sin embargo, y más allá de compartir estas aseveraciones, este índice tiene una curiosa propiedad: atribuye una mayor importancia a los cambios distributivos que tienen lugar en el centro (moda) de la distribución (en nuestro caso, meses de demanda media anual), y da un peso simétrico a los cambios en los extremos (i.e. meses de mayor y menor demanda). En este sentido, parecería razonable no estar necesariamente de acuerdo con este funcionamiento automático. Como alternativa es posible que los investigadores aprecien las ventajas de medidas que son especialmente sensibles a los cambios que ocurren 
en la parte baja de la distribución y, por tanto, en nuestro caso a los meses de menor demanda. Entre estos indices «progresivos» han gozaso de atención los índices de la familia de Theil (Theil, 1967), que responderían a la formulación siguiente:

$$
T(\beta)=\frac{1}{\beta(\beta-1)} \sum_{i} p_{i}\left[\left(\frac{y_{i}}{\mu}\right)^{\beta}-1\right] \quad \beta \neq 0,1
$$

El parámetro $\beta$ precisamente refleja la sensibilidad de la medida ante el lugar donde se producen los cambios distributivos. Específicamente, cuanto menor (mayor) es el valor del parámetro mayor (menor) es la progresividad de la medida. Por ejemplo, para valores de $\beta$ superiores a 2 la medida sólo parece mostrar sensibilidad ante la igualación entre los meses de mayor demanda.

De entre dicha familia, los índices particulares que han gozado de mayor atención son el $\mathrm{T}(0)$ y $\mathrm{T}(1)$, cuyas expresiones algebraicas serían:

$$
\begin{gathered}
T(\beta=0)=-\sum_{i} p_{p} \log \left(\frac{\mu}{y_{i}}\right) \\
T(\beta=1)=\sum_{i} p_{p}\left(\frac{y_{i}}{\mu}\right) \log \left(\frac{y_{i}}{\mu}\right)
\end{gathered}
$$

Por otra parte, quizás nos interesa asimismo una medida que no sea especialmente sensible a ninguna parte de la distribución y que, por tanto, se comporte de manera neutral. En este sentido, una buena medida candidata podría ser el Coeficiente de Variación $(\mathrm{CV})$, que, como se sabe, no es más que la división de la desviación típica por la media y que acierta a cumplir los axiomas básicos exigidos a toda medida satisfactoria. De hecho, si lo elevamos al cuadrado y dividimos por la mitad dicha medida es ordinalmente equivalente a $\mathrm{T}(2)$.

\section{MEDICIÓN DE LA ESTACIONALIDAD TURÍSTICA EN LAS PROVINCIAS ESPAÑOLAS, 1999-2012}

Los datos básicos para el análisis provienen de la extracción de información a partir de la Encuesta de Ocupación Hotelera, realizada periódicamente por el INE. La variable de demanda turística seleccionada han sido las pernoctaciones hoteleras y cubren el período 1999-2012, máximo disponible en la actualidad. De esta manera, el período nos permite examinar la evolución a medio plazo de la estacionalidad en España, más allá de los posibles vaivenes anuales. En este sentido, estas pernoctaciones suponen la mayoría de las pernoctaciones regladas producidas en las provincias. Se han seleccionado todas las provincias españolas, exceptuando Ceuta y Melilla por su limitado tamaño. En particular, se pretende realizar un análisis global de la concentración temporal de la actividad 
turística en España a nivel territorial, sin detallar en exceso el análisis para cada una de ellas, en primer lugar, para salvar espacio y, en segundo lugar, para concentrarnos en las observaciones de patrones generales ${ }^{3}$.

\subsection{Análisis 2012}

En el Cuadro 1 se suministran los resultados obtenidos para el 2012 cuando se aplican las cuatro medidas sintéticas antes revisadas, esto es, el Gini, el T(0), el T(1) y el C.V. Las provincias aparecen ordenadas de forma descendente en función del coeficiente de variación, en particular. Se puede observar, por ejemplo, la amplia diversidad existente a nivel territorial. En particular, la concentración mensual se mueve desde los registros mínimos (casi distribuciones uniformes) de las provincias canarias o de Madrid, con cifras próximas a cero del Gini y, en los tres casos, provincias de elevada demanda hotelera, a los elevados registros que exhiben las Baleares o provincias de la costa catalana (exceptuando Barcelona). De hecho, se observa que los extremos del ranking vienen protagonizados típicamente también por provincias de elevada demanda hotelera.

Por otra parte, obsérvese que parecería que las provincias que lideran el ranking típicamente son costeras, o si se quiere, especializadas fundamentalmente en sol y playa, aunque no sólo. Sin embargo, no parece que esta característica sea unánimemente determinante de una mayor concentración de la actividad, como demuestran los casos de Cádiz, Málaga, Murcia, Alicante y, sobretodo, Canarias. Habría, pues, algo más que el producto que ayudaría a explicar la estacionalidad concreta, y sus diferencias territoriales, como podría ser el caso de la temperatura (en el caso de Canarias no solo la media anual sino su reducida dispersión mensual, por ejemplo), los mercados o incluso la política.

Asimismo, parece que las provincias interiores tienen menos problemas en este sentido. La parte baja del ranking, y media, viene protagonizada, en efecto, masivamente por este tipo de provincias, típicamente de menor demanda hotelera. De hecho, no se ubica ninguna de ellas en los primeros lugares del ranking ${ }^{4}$.

En todo caso, y dado que las diferentes medidas pueden dar resultados distintos, hemos calculado los coeficientes de correlación de Pearson entre las diferentes medidas para años seleccionados del período sintetizados en el Cuadro 2. Se observa, de manera resumida, que las correlaciones son elevadas, aunque no perfectas. De esta manera, y aunque en términos generales no hay cambios significativos en los rankings provinciales en base al uso de medidas concretas, si que se da alguna discrepancia en casos concretos.

3 En Duro (2008) se realizaba un análisis parecido aplicado a las Comunidades Autónomas españolas y que finalizaba en el ejercicio de 2005. Lopez y López (2006) también han analizado la concentración de la actividad pero asociada a la oferta turística y también a nivel de CCAA. Fernández y Mayorga (2008) analizan, asimismo, la concentración de la actividad turística en las provincias de la Costa del Sol bajo un enfoque de desigualdad similar.

4 Veremos de una manera más sistemática en la sección anterior las correlaciones entre la estacionalidad y una seria de factores potencialmente significativos 
Cuadro 1

CONCENTRACIÓN MENSUAL DE LA DEMANDA

HOTELERA PROVINCIAS ESPAÑOLAS, 2012

\begin{tabular}{|c|c|c|c|c|c|}
\hline & C.V. & Theil-0 & Theil-1 & Gini & P. Hot \\
\hline Balears, Illes & 0,8824 & 0,7210 & 0,4402 & 0,4906 & 54.242 \\
\hline Tarragona & 0,8692 & 0,5708 & 0,4029 & 0,4800 & 9.717 \\
\hline Girona & 0,7596 & 0,3473 & 0,2867 & 0,4194 & 10.591 \\
\hline Huelva & 0,7097 & 0,2968 & 0,2508 & 0,3934 & 3.327 \\
\hline Castellón/Castelló & 0,6762 & 0,2450 & 0,2203 & 0,3711 & 3.238 \\
\hline Almería & 0,6738 & 0,2223 & 0,2098 & 0,3619 & 4.106 \\
\hline Pontevedra & 0,6064 & 0,1618 & 0,1635 & 0,3181 & 2.508 \\
\hline Cantabria & 0,6026 & 0,1798 & 0,1702 & 0,3254 & 2.265 \\
\hline Cádiz & 0,5976 & 0,2136 & 0,1830 & 0,3367 & 6.174 \\
\hline Asturias & 0,5454 & 0,1396 & 0,1372 & 0,2922 & 2.805 \\
\hline Lugo & 0,4713 & 0,1138 & 0,1084 & 0,2612 & 785 \\
\hline Huesca & 0,4460 & 0,0907 & 0,0907 & 0,2256 & 1.443 \\
\hline Málaga & 0,4343 & 0,1091 & 0,0983 & 0,2469 & 16.063 \\
\hline Coruña, A & 0,4079 & 0,0785 & 0,0786 & 0,2220 & 2.859 \\
\hline Lleida & 0,3677 & 0,0683 & 0,0661 & 0,2029 & 1.646 \\
\hline Barcelona & 0,3662 & 0,0728 & 0,0684 & 0,2085 & 26.423 \\
\hline Gipuzkoa & 0,3627 & 0,0692 & 0,0660 & 0,2055 & 1.779 \\
\hline Murcia & 0,3383 & 0,0592 & 0,0570 & 0,1911 & 2.537 \\
\hline Alicante/Alacant & 0,3352 & 0,0559 & 0,0551 & 0,1877 & 14.930 \\
\hline Navarra & 0,3298 & 0,0592 & 0,0557 & 0,1870 & 1.491 \\
\hline Valencia/València & 0,3250 & 0,0548 & 0,0528 & 0,1840 & 5.813 \\
\hline Burgos & 0,3185 & 0,0539 & 0,0512 & 0,1780 & 1.063 \\
\hline Palencia & 0,2940 & 0,0442 & 0,0430 & 0,1647 & 303 \\
\hline Soria & 0,2931 & 0,0441 & 0,0427 & 0,1635 & 336 \\
\hline Bizkaia & 0,2812 & 0,0394 & 0,0389 & 0,1569 & 2.205 \\
\hline Teruel & 0,2803 & 0,0364 & 0,0372 & 0,1502 & 562 \\
\hline Segovia & 0,2706 & 0,0380 & 0,0369 & 0,1541 & 563 \\
\hline Rioja, La & 0,2648 & 0,0401 & 0,0370 & 0,1489 & 879 \\
\hline Ourense & 0,2590 & 0,0356 & 0,0340 & 0,1415 & 491 \\
\hline Cáceres & 0,2586 & 0,0383 & 0,0353 & 0,1431 & 1.054 \\
\hline Zamora & 0,2559 & 0,0326 & 0,0323 & 0,1405 & 350 \\
\hline Ávila & 0,2543 & 0,0339 & 0,0327 & 0,1436 & 424 \\
\hline León & 0,2491 & 0,0336 & 0,0319 & 0,1396 & 1.066 \\
\hline Araba/Álava & 0,2475 & 0,0288 & 0,0293 & 0,1339 & 651 \\
\hline Albacete & 0,2440 & 0,0364 & 0,0325 & 0,1321 & 617 \\
\hline Salamanca & 0,2405 & 0,0330 & 0,0305 & 0,1352 & 1.456 \\
\hline Cuenca & 0,2281 & 0,0272 & 0,0264 & 0,1293 & 438 \\
\hline Sevilla & 0,2194 & 0,0241 & 0,0239 & 0,1245 & 4.491 \\
\hline Badajoz & 0,2189 & 0,0269 & 0,0252 & 0,1229 & 1.037 \\
\hline Jaén & 0,2095 & 0,0232 & 0,0224 & 0,1180 & 772 \\
\hline Córdoba & 0,2072 & 0,0211 & 0,0212 & 0,1166 & 1.609 \\
\hline Zaragoza & 0,1762 & 0,0171 & 0,0162 & 0,0945 & 1.960 \\
\hline Granada & 0,1572 & 0,0133 & 0,0128 & 0,0881 & 4.640 \\
\hline Toledo & 0,1552 & 0,0127 & 0,0123 & 0,0883 & 1.013 \\
\hline Ciudad Real & 0,1496 & 0,0128 & 0,0119 & 0,0785 & 543 \\
\hline Valladolid & 0,1401 & 0,0102 & 0,0100 & 0,0797 & 909 \\
\hline Guadalajara & 0,1353 & 0,0099 & 0,0095 & 0,0750 & 363 \\
\hline Palmas, Las & 0,1151 & 0,0064 & 0,0065 & 0,0629 & 33.661 \\
\hline Madrid & 0,1084 & 0,0059 & 0,0059 & 0,0614 & 18.626 \\
\hline Santa Cruz de Tenerife & 0,0744 & 0,0028 & 0,0028 & 0,0419 & 23.555 \\
\hline TOTAL NACIONAL & 0,4037 & 0,0831 & 0,0804 & 0,2273 & 280.382 \\
\hline
\end{tabular}

Nota: ranking provincial en función del C.V. Pernoctaciones Hoteleras figuran en miles.

Fuente: elaboración propia a partir de datos de la Encuesta de Ocupación Hotelera (EOH), INE. 


\section{Mapa 1 \\ PROVINCIAS DISTRIBUIDAS SEGÚN NIVEL DE CONCENTRACIÓN MENSUAL DE LA DEMANDA HOTELERA, 2012}

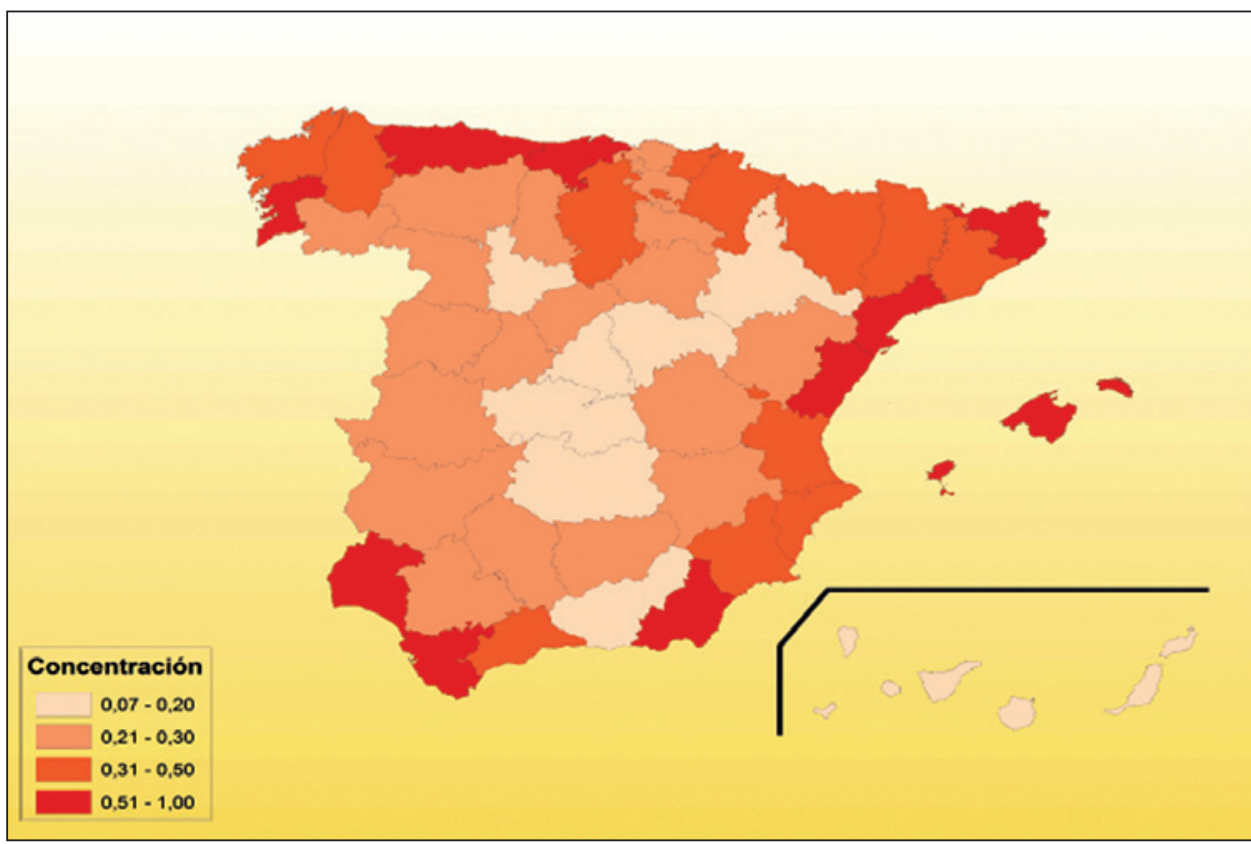

Nota: se ha utilizado el CV como referencia para la medición de la concentración mensual. Fuente: elaboración propia a partir de datos de la Encuesta de Ocupación Hotelera (EOH), INE.

Cuadro 2

COEFICIENTES DE CORRELACIÓN PARCIAL ENTRE ÍNDICES DE CONCENTRACIÓN MENSUAL, AÑOS SELECCIONADOS PERÍODO

\begin{tabular}{|l|cccc|}
\hline & $\mathbf{1 9 9 9}$ & $\mathbf{2 0 0 4}$ & $\mathbf{2 0 0 8}$ & $\mathbf{2 0 1 2}$ \\
\hline CV versus Theil-0 & 0,8844 & 0,9109 & 0,9147 & 0,8965 \\
CV versus Theil-1 & 0,9477 & 0,9577 & 0,9563 & 0,9574 \\
CV versus Gini & 0,9975 & 0,9981 & 0,9993 & 0,9989 \\
Theil-0 versus Theil-1 & 0,9833 & 0,9892 & 0,9914 & 0,9823 \\
Theil-0 versus Gini & 0,8957 & 0,9219 & 0,9182 & 0,9022 \\
Theil-1 versus Gini & 0,9536 & 0,9637 & 0,9580 & 0,9600 \\
\hline
\end{tabular}

Fuente: Elaboración propia a partir de datos de la Encuesta de Ocupación Hotelera (EOH), INE. 


\subsection{Evolución}

En lo que respecta a la variabilidad temporal, y aunque a nivel global se produce un aumento de la concentración en el período agregado, que se aproxima al 5\% en el caso de Gini y al 5.7\% para el CV, se observa una gran variabilidad territorial. Típicamente, la concentración parecería haber crecido en provincias de mayor desequilibrio mensual. En este sentido, el patrón trazado por las Baleares es especialmente ilustrativo. También habría que destacar el creciente desequilibrio registrado en provincias costeras andaluzas como Huelva o Almería. De hecho, a partir de estos resultados agregados podemos hacer algunos ejercicios ilustrativos de naturaleza sintética. Por ejemplo, en este escenario tiene sentido testar si se produce una hipótesis de convergencia-divergencia en la concentración en estos años. Esto es, si las provincias de menor concentración inicial experimentan un crecimiento mayor o menor que la media. Este es el llamado ejercicio de $\beta$-convergencia (Barro y Sala-i-Martin, 1992) y sus resultados se ofrecen a continuación:

$$
\operatorname{Ln}(\mathrm{Ci}, \mathrm{t})-\operatorname{Ln}(\mathrm{Ci}, \mathrm{t}-1)=-0.099-0.0602 * \operatorname{Ln}(\mathrm{Ci}, \mathrm{t}-1)
$$

(1.18) (0.92)

Nota: estadísticos t-student entre paréntesis.

Se observa, pues, que no hay un patrón global evolutivo en el sentido anterior, negando, pues, la significatividad de cualquier elemento de automaticidad en la evolución.

Para un mayor detalle de las evoluciones, puede ser, asimismo, útil elaborar una estructuración ad-hoc de la evolución de concentración en función de diversos parámetros. Por ejemplo, uno de ellos sería el nivel inicial, como hemos dicho. De esta manera, podríamos dibujar cuatro posibles estadios en función de la combinación de concentración relativa elevada-baja y/o crecimiento relativo elevado-alto (diagrama 1). En la peor de las situaciones encontraríamos, en particular, al grueso de provincias andaluzas costeras y a las Baleares, descritas por una concentración de la demanda comparativamente elevada y un crecimiento superior al de toda España. En todos los casos, se trata de provincias predominantemente especializadas en sol y playa. En contraste, las provincias catalanas con una especialización «parecida» a las anteriores y las cantábricas habrían evolucionado mejor. En el cuadrante inferior izquierdo se encuentran las provincias de concentración baja y evolución favorable, donde se encuentran provincias de demanda significada, como Barcelona, Sevilla, Madrid o Sta Cruz de Tenerife. Finalmente entre el grupo de concentración baja pero empeoramiento significado hay que destacar el retroceso en provincias de elevada especialización turística como Alicante, Valencia o Las Palmas.

En todo caso, y cuando abordamos la evolución concreta de la concentración mensual de la actividad en base a cada índice (cuadro 3), se observan discrepancias cardinales. Incluso, en algunos casos, como los trazados por las provincias de Tarragona, Asturias, Lugo o Segovia se observan discrepancias en el signo de la evolución. Por 
ejemplo, en el caso de la provincias de Tarragona la concentración mensual, en base al C.V, habría aumentado levemente, mientras que la señal proporcionada por el $\mathrm{T}(0)$ es la de una clara reducción. En este sentido, pues, y aunque en términos de ordenación no se dibujen diferencias muy relevantes, a la hora de evaluar las evoluciones concretas se ha de prestar atención a las medidas utilizadas y a las provincias concretas analizadas.

\section{Diagrama 1 \\ SITUACIONES PROVINCIALES NIVELES DE CONCENTRACIÓN Y PATRÓN TEMPORAL 1999-2012}

\begin{tabular}{|l|l|}
\hline Concentración alta-Crec. Bajo & Concentración alta-Crec. Alto \\
\hline $\begin{array}{l}\text { Tarragona, Girona, Castellón, Pontevedra, Cantabria, Astu- } \\
\text { rias, Lugo, Huesca, Coruña }\end{array}$ & $\begin{array}{l}\text { Baleares, Huelva, Almería, Cá- } \\
\text { diz, Málaga }\end{array}$ \\
\hline Concentración baja.Crec. bajo & Concentración baja-Crec. alto \\
\hline $\begin{array}{l}\text { Lleida, Barcelona, Guipúzcoa, Murcia, Navarra, Bizcaia, Teruel, } \\
\text { Segovia, Rioja, Ourense, Cáceres, Zamora, Ávila, León, Sala- } \\
\text { manca, Cuenca, Sevilla, Badajoz, Córdoba, Zaragoza, Granada, } \\
\text { Toledo, CR, Valladolid, Guadalajara, Madrid, Sta Cruz }\end{array}$ & $\begin{array}{l}\text { Alicante, Valencia, Burgos, Pa- } \\
\text { lencia, Soria, Alava, Albacete, } \\
\text { Jaén, Las Palmas }\end{array}$ \\
\hline
\end{tabular}

Fuente: Elaboración propia a partir de datos de la Encuesta de Ocupación Hotelera (EOH), INE.

Nota: hemos tomado el C.V como referencia. Los términos altos y bajo están referenciados en términos relativos a la media nacional.

Como análisis complementario al anterior, hemos reproducido la evolución trazada por la concentración mensual para el último subperíodo 2008-2012 (cuadro 4). Como es conocido, estos años vienen presididos por la actual crisis económica e incluyen, por ejemplo, en el ámbito turístico, la gran crisis del 2009 y la recuperación posterior. En este sentido, nuevamente se constata el aumento a nivel nacional de la concentración, que llega incluso al 18\% según los índices de Theil. Por provincias se observan crecimientos destacados a nivel global, y en particular en territorios de alta demanda, como Balears, Tarragona, Girona, Málaga, Alicante o Valencia. En contraste, hay que subrayar la favorable evolución en las provincias canarias, o el menor aumento comparativo producido en la provincia de Barcelona. En el mapa 2 se ha sintetizado de manera visual el crecimiento de las provincias, a partir del CV. Así, se observa, por ejemplo, aproximadamente una dinámica de crecimiento diferencial de la concentración en las provincias situada en un eje que va de Sur a Nordeste, y que comienza en Cádiz y acaba en las Baleares (con la provincia de Granada como gran excepción). Nuevamente habría que ir con precaución en orden a evaluar los aumentos concretos según los índices, dadas las diferencias detectadas entre ellos. Destaca, en este sentido, por ejemplo, el caso de la provincia de Huesca, donde según el CV la concentración habría crecido cuando en base a $\mathrm{T}(0)$ o el Gini ésta habría disminuido con claridad. Cualitativamente también es de destacar el caso de Las Palmas, donde la mayoría de medidas señalan un descenso significativo mientras que el Gini indica una reducción muy poco relevante. 
Cuadro 3

VARIACIÓN CONCENTRACIÓN MENSUAL DE LA DEMANDA HOTELERA PROVINCIAS ESPAÑOLAS PERÍODO 1999-2012

\begin{tabular}{|c|c|c|c|c|}
\hline & CV & $\mathbf{T}(\mathbf{o})$ & $\mathbf{T}(\mathbf{1})$ & G \\
\hline Balears, Illes & $34,6 \%$ & $137,4 \%$ & $85,4 \%$ & $34,4 \%$ \\
\hline Tarragona & $1,1 \%$ & $-13,6 \%$ & $-4,3 \%$ & $0,4 \%$ \\
\hline Girona & $-11,2 \%$ & $-29,7 \%$ & $-23,3 \%$ & $-10,9 \%$ \\
\hline Huelva & $48,4 \%$ & $127,9 \%$ & $114,7 \%$ & $45,0 \%$ \\
\hline Castellón/Castelló & $4,8 \%$ & $16,6 \%$ & $12,1 \%$ & $5,7 \%$ \\
\hline Almería & $61,7 \%$ & $154,2 \%$ & $147,2 \%$ & $54,8 \%$ \\
\hline Pontevedra & $-8,8 \%$ & $-26,2 \%$ & $-21,0 \%$ & $-11,7 \%$ \\
\hline Cantabria & $-13,5 \%$ & $-22,3 \%$ & $-22,6 \%$ & $-11,7 \%$ \\
\hline Cádiz & $25,4 \%$ & $68,5 \%$ & $58,8 \%$ & $26,0 \%$ \\
\hline Asturias & $-4,2 \%$ & $1,0 \%$ & $-3,4 \%$ & $-0,2 \%$ \\
\hline Lugo & $-5,9 \%$ & $-0,2 \%$ & $-4,6 \%$ & $0,3 \%$ \\
\hline Huesca & $-11,3 \%$ & $-27,7 \%$ & $-22,5 \%$ & $-11,6 \%$ \\
\hline Málaga & $31,9 \%$ & $85,4 \%$ & $77,1 \%$ & $31,9 \%$ \\
\hline Coruña, A & $-2,8 \%$ & $-9,4 \%$ & $-7,7 \%$ & $-5,1 \%$ \\
\hline Lleida & $-15,2 \%$ & $-21,8 \%$ & $-23,4 \%$ & $-8,0 \%$ \\
\hline Barcelona & $-12,9 \%$ & $-24,5 \%$ & $-24,0 \%$ & $-12,8 \%$ \\
\hline Gipuzkoa & $-10,1 \%$ & $-14,6 \%$ & $-16,5 \%$ & $-8,9 \%$ \\
\hline Murcia & $-0,2 \%$ & $-7,2 \%$ & $-3,9 \%$ & $-1,0 \%$ \\
\hline Alicante/Alacant & $44,4 \%$ & $108,6 \%$ & $106,7 \%$ & $43,1 \%$ \\
\hline Navarra & $-10,3 \%$ & $-15,2 \%$ & $-17,0 \%$ & $-9,8 \%$ \\
\hline Valencia/València & $55,4 \%$ & $140,3 \%$ & $138,3 \%$ & $57,7 \%$ \\
\hline Burgos & $-9,3 \%$ & $-14,2 \%$ & $-15,9 \%$ & $-9,7 \%$ \\
\hline Palencia & $-14,7 \%$ & $-25,6 \%$ & $-26,3 \%$ & $-15,3 \%$ \\
\hline Soria & $-18,2 \%$ & $-27,8 \%$ & $-29,7 \%$ & $-14,8 \%$ \\
\hline Bizkaia & $73,5 \%$ & $187,9 \%$ & $191,6 \%$ & $72,5 \%$ \\
\hline Teruel & $-19,5 \%$ & $-30,0 \%$ & $-32,0 \%$ & $-14,4 \%$ \\
\hline Segovia & $-0,9 \%$ & $-14,8 \%$ & $-7,5 \%$ & $2,0 \%$ \\
\hline Rioja, La & $-8,1 \%$ & $-13,9 \%$ & $-14,0 \%$ & $-7,9 \%$ \\
\hline Ourense & $-40,2 \%$ & $-57,0 \%$ & $-60,4 \%$ & $-38,5 \%$ \\
\hline Cáceres & $-1,5 \%$ & $-7,9 \%$ & $-5,2 \%$ & $-2,7 \%$ \\
\hline Zamora & $-29,2 \%$ & $-49,6 \%$ & $-48,8 \%$ & $-27,2 \%$ \\
\hline Ávila & $-24,9 \%$ & $-43,5 \%$ & $-43,0 \%$ & $-24,6 \%$ \\
\hline León & $-26,0 \%$ & $-41,6 \%$ & $-42,8 \%$ & $-25,3 \%$ \\
\hline Araba/Álava & $35,7 \%$ & $77,8 \%$ & $79,7 \%$ & $31,3 \%$ \\
\hline Albacete & $33,7 \%$ & $124,9 \%$ & $99,0 \%$ & $29,6 \%$ \\
\hline Salamanca & $-16,1 \%$ & $-30,3 \%$ & $-29,5 \%$ & $-14,4 \%$ \\
\hline Cuenca & $-31,4 \%$ & $-46,9 \%$ & $-49,4 \%$ & $-27,0 \%$ \\
\hline Sevilla & $-0,6 \%$ & $-9,8 \%$ & $-5,5 \%$ & $-0,1 \%$ \\
\hline Badajoz & $-3,9 \%$ & $-9,6 \%$ & $-8,4 \%$ & $-4,0 \%$ \\
\hline Jaén & $22,2 \%$ & $44,7 \%$ & $46,6 \%$ & $22,2 \%$ \\
\hline Córdoba & $-16,0 \%$ & $-37,2 \%$ & $-33,2 \%$ & $-17,2 \%$ \\
\hline Zaragoza & $-21,6 \%$ & $-46,1 \%$ & $-42,1 \%$ & $-22,0 \%$ \\
\hline Granada & $-13,5 \%$ & $-23,6 \%$ & $-24,2 \%$ & $-15,0 \%$ \\
\hline Toledo & $-26,2 \%$ & $-45,4 \%$ & $-45,2 \%$ & $-26,4 \%$ \\
\hline Ciudad Real & $-22,0 \%$ & $-37,6 \%$ & $-38,3 \%$ & $-27,1 \%$ \\
\hline Valladolid & $-14,7 \%$ & $-30,3 \%$ & $-28,7 \%$ & $-14,1 \%$ \\
\hline Guadalajara & $-17,3 \%$ & $-30,8 \%$ & $-31,1 \%$ & $-17,5 \%$ \\
\hline Palmas, Las & $49,8 \%$ & $120,4 \%$ & $122,0 \%$ & $46,4 \%$ \\
\hline Madrid & $-7,7 \%$ & $-15,6 \%$ & $-15,2 \%$ & $-7,3 \%$ \\
\hline Santa Cruz de Tenerife & $-21,8 \%$ & $-36,0 \%$ & $-37,4 \%$ & $-18,4 \%$ \\
\hline TOTAL NACIONAL & $5,7 \%$ & $9,1 \%$ & $5,7 \%$ & $4,9 \%$ \\
\hline
\end{tabular}

Fuente: Elaboración propia a partir de datos de la Encuesta de Ocupación Hotelera (EOH), INE. 


\section{Cuadro 4}

VARIACIÓN CONCENTRACIÓN MENSUAL DE LA DEMANDA HOTELERA PROVINCIAS ESPAÑOLAS SUBPERÍODO AGREGADO 2008-2012

\begin{tabular}{|c|c|c|c|c|}
\hline & $\mathrm{CV}$ & $\mathbf{T}(\mathbf{0})$ & $\mathbf{T}(\mathbf{1})$ & $\mathbf{G}$ \\
\hline Balears, Illes & $8,6 \%$ & $37,5 \%$ & $21,4 \%$ & $7,8 \%$ \\
\hline Tarragona & $6,3 \%$ & $21,3 \%$ & $14,4 \%$ & $5,5 \%$ \\
\hline Girona & $13,5 \%$ & $24,7 \%$ & $25,1 \%$ & $12,6 \%$ \\
\hline Huelva & $18,7 \%$ & $47,0 \%$ & $40,9 \%$ & $17,9 \%$ \\
\hline Castellón/Castelló & $31,3 \%$ & $81,2 \%$ & $71,8 \%$ & $29,9 \%$ \\
\hline Almería & $44,4 \%$ & $113,4 \%$ & $104,7 \%$ & $42,7 \%$ \\
\hline Pontevedra & $13,0 \%$ & $25,6 \%$ & $25,3 \%$ & $11,4 \%$ \\
\hline Cantabria & $8,0 \%$ & $17,3 \%$ & $16,1 \%$ & $6,9 \%$ \\
\hline Cádiz & $13,5 \%$ & $34,8 \%$ & $30,0 \%$ & $13,5 \%$ \\
\hline Asturias & $19,1 \%$ & $43,6 \%$ & $40,7 \%$ & $18,2 \%$ \\
\hline Lugo & $24,4 \%$ & $69,0 \%$ & $60,2 \%$ & $27,5 \%$ \\
\hline Huesca & $5,4 \%$ & $-7,6 \%$ & $0,3 \%$ & $-5,0 \%$ \\
\hline Málaga & $19,9 \%$ & $55,2 \%$ & $47,9 \%$ & $19,7 \%$ \\
\hline Coruña, A & $36,9 \%$ & $85,6 \%$ & $84,5 \%$ & $36,1 \%$ \\
\hline Lleida & $-7,7 \%$ & $-20,8 \%$ & $-17,7 \%$ & $-9,1 \%$ \\
\hline Barcelona & $4,5 \%$ & $12,5 \%$ & $10,6 \%$ & $4,6 \%$ \\
\hline Gipuzkoa & $19,2 \%$ & $54,0 \%$ & $46,9 \%$ & $20,9 \%$ \\
\hline Murcia & $18,3 \%$ & $22,0 \%$ & $30,6 \%$ & $18,9 \%$ \\
\hline Alicante/Alacant & $26,5 \%$ & $61,2 \%$ & $59,7 \%$ & $25,6 \%$ \\
\hline Navarra & $13,2 \%$ & $26,2 \%$ & $26,6 \%$ & $12,5 \%$ \\
\hline Valencia/València & $36,3 \%$ & $84,3 \%$ & $83,6 \%$ & $38,0 \%$ \\
\hline Burgos & $9,9 \%$ & $22,1 \%$ & $21,0 \%$ & $9,2 \%$ \\
\hline Palencia & $16,9 \%$ & $33,2 \%$ & $34,0 \%$ & $16,6 \%$ \\
\hline Soria & $-9,1 \%$ & $-17,1 \%$ & $-16,6 \%$ & $-7,3 \%$ \\
\hline Bizkaia & $54,2 \%$ & $129,9 \%$ & $132,0 \%$ & $52,9 \%$ \\
\hline Teruel & $4,5 \%$ & $11,2 \%$ & $10,0 \%$ & $5,4 \%$ \\
\hline Segovia & $11,1 \%$ & $23,2 \%$ & $23,2 \%$ & $12,3 \%$ \\
\hline Rioja, La & $10,4 \%$ & $29,8 \%$ & $25,3 \%$ & $8,9 \%$ \\
\hline Ourense & $31,5 \%$ & $73,0 \%$ & $71,8 \%$ & $31,4 \%$ \\
\hline Cáceres & $15,3 \%$ & $38,9 \%$ & $35,3 \%$ & $12,4 \%$ \\
\hline Zamora & $-13,9 \%$ & $-24,8 \%$ & $-25,2 \%$ & $-14,1 \%$ \\
\hline Ávila & $-5,5 \%$ & $-10,4 \%$ & $-10,2 \%$ & $-3,6 \%$ \\
\hline León & $1,4 \%$ & $5,6 \%$ & $4,4 \%$ & $0,9 \%$ \\
\hline Araba/Álava & $43,1 \%$ & $91,2 \%$ & $96,5 \%$ & $36,5 \%$ \\
\hline Albacete & $23,6 \%$ & $54,3 \%$ & $52,9 \%$ & $27,0 \%$ \\
\hline Salamanca & $8,0 \%$ & $13,5 \%$ & $15,1 \%$ & $9,9 \%$ \\
\hline Cuenca & $5,7 \%$ & $10,7 \%$ & $11,3 \%$ & $7,2 \%$ \\
\hline Sevilla & $15,4 \%$ & $32,0 \%$ & $32,3 \%$ & $14,9 \%$ \\
\hline Badajoz & $18,4 \%$ & $40,3 \%$ & $39,8 \%$ & $21,0 \%$ \\
\hline Jaén & $15,2 \%$ & $26,2 \%$ & $29,3 \%$ & $18,0 \%$ \\
\hline Córdoba & $3,0 \%$ & $2,4 \%$ & $4,2 \%$ & $2,7 \%$ \\
\hline Zaragoza & $-41,4 \%$ & $-64,5 \%$ & $-64,7 \%$ & $-44,7 \%$ \\
\hline Granada & $3,8 \%$ & $7,8 \%$ & $7,8 \%$ & $4,7 \%$ \\
\hline Toledo & $0,0 \%$ & $-0,6 \%$ & $-0,3 \%$ & $0,6 \%$ \\
\hline Ciudad Real & $-2,4 \%$ & $-0,9 \%$ & $-2,9 \%$ & $-4,7 \%$ \\
\hline Valladolid & $7,5 \%$ & $17,4 \%$ & $16,5 \%$ & $9,6 \%$ \\
\hline Guadalajara & $-2,9 \%$ & $-4,7 \%$ & $-5,2 \%$ & $-4,2 \%$ \\
\hline Palmas, Las & $-8,9 \%$ & $-10,8 \%$ & $-13,9 \%$ & $-0,4 \%$ \\
\hline Madrid & $15,0 \%$ & $31,6 \%$ & $31,9 \%$ & $16,1 \%$ \\
\hline Santa Cruz de Tenerife & $-24,8 \%$ & $-40,1 \%$ & $-41,8 \%$ & $-23,7 \%$ \\
\hline TOTAL NACIONAL & $8,6 \%$ & $18,1 \%$ & $17,8 \%$ & $8,1 \%$ \\
\hline
\end{tabular}

Fuente: Elaboración propia a partir de datos de la Encuesta de Ocupación Hotelera (EOH), INE. 


\section{Mapa 2 \\ PROVINCIAS SEGÚN CRECIMIENTO DE LA CONCENTRACIÓN MENSUAL DE LA DEMANDA HOTELERA, 1999-2012}

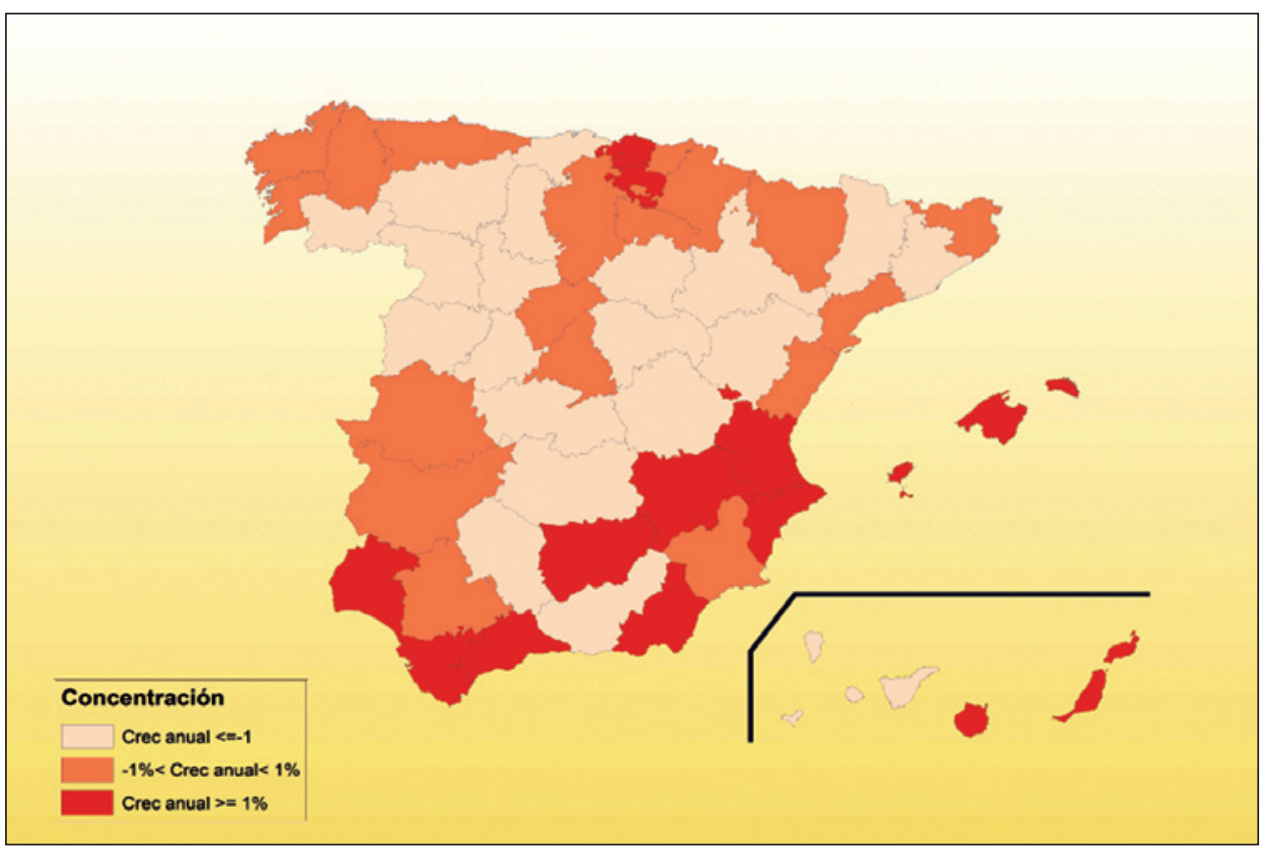

Nota: se ha utilizado el CV como referencia para la medición del crecimiento de la concentración mensual. Fuente: Elaboración propia a partir de datos de la Encuesta de Ocupación Hotelera (EOH), INE.

\subsection{Resultados medición concentración sobre una base cuatrimestral}

Adicionalmente, se ha realizado un análisis de la concentración turística provincial sobre una base cuatrimestral. Así las cosas, se han establecido ad-hoc tres períodos de igual extensión y peso que globalmente parecen razonables como indicativos de temporadas turísticas, permitiendo, además, salvar el desajuste de los efectos calendario motivados por festividades como la de Semana Santa en la demanda turística territorial. En términos empíricos, los índices sobre esta base arrojan evoluciones más uniformes que los surgidos del análisis mensual. En el Cuadro adjuntado, y para salvar espacio, sólo se exhiben los resultados asociados al uso del C.V. que, como se indicó en la sección anterior, es neutro en referencia a la sensibilidad distributiva y, además, comparte con el Gini su limitada sensibilidad ante valores extremos (i.e. evolución más uniforme).

Antes de todo, comentar que si se computa el coeficiente de correlación provincial entre las medidas sobre una base mensual (anteriormente analizadas) y cuatrimestral se observan valores elevados. Por ejemplo, para 1999 este coeficiente alcanza un valor del $0.92,0.91$ en 2004 y 2008 y 0.93 en 2012. De hecho, una mirada al ranking establecido, por ejemplo, para 2012, revela discrepancias no elevadas. Las cinco regiones 
Cuadro 5

VARIACIÓN CONCENTRACIÓN CUATRIMESTRAL DE LA DEMANDA HOTELERA PROVINCIAS ESPAÑOLAS SUBPERÍODO AGREGADO 2008-2012

\begin{tabular}{|c|c|c|c|c|c|c|}
\hline & 1999 & 2004 & 2008 & 2012 & $\begin{array}{c}\text { Var, } \\
\text { 1999-2012 }\end{array}$ & $\begin{array}{c}\text { Var. } \\
\text { 2008-2012 }\end{array}$ \\
\hline Balears, Illes & 1,1402 & 1,2225 & 1,2423 & 1,2933 & $13,4 \%$ & $4,1 \%$ \\
\hline Tarragona & 1,2731 & 1,2106 & 1,2477 & 1,2632 & $-0,8 \%$ & $1,2 \%$ \\
\hline Girona & 1,2376 & 1,1397 & 1,1088 & 1,1567 & $-6,5 \%$ & $4,3 \%$ \\
\hline Huelva & 0,9648 & 1,0409 & 1,0532 & 1,0897 & $12,9 \%$ & $3,5 \%$ \\
\hline Castellón/Castelló & 1,0715 & 1,0303 & 1,0099 & 1,0727 & $0,1 \%$ & $6,2 \%$ \\
\hline Cádiz & 1,0050 & 1,0262 & 1,0174 & 1,0652 & $6,0 \%$ & $4,7 \%$ \\
\hline Cantabria & 1,0978 & 1,0883 & 1,0546 & 1,0629 & $-3,2 \%$ & $0,8 \%$ \\
\hline Pontevedra & 1,1140 & 1,0913 & 1,0279 & 1,0533 & $-5,5 \%$ & $2,5 \%$ \\
\hline Lugo & 1,0230 & 0,9969 & 0,9548 & 1,0203 & $-0,3 \%$ & $6,9 \%$ \\
\hline Asturias & 1,0443 & 1,0305 & 0,9807 & 1,0195 & $-2,4 \%$ & $4,0 \%$ \\
\hline Almería & 0,9706 & 0,9650 & 0,9659 & 1,0183 & $4,9 \%$ & $5,4 \%$ \\
\hline Coruña, A & 0,9905 & 0,9611 & 0,9187 & 0,9825 & $-0,8 \%$ & $6,9 \%$ \\
\hline Málaga & 0,9354 & 0,9219 & 0,9474 & 0,9760 & $4,3 \%$ & $3,0 \%$ \\
\hline Gipuzkoa & 0,9775 & 0,9626 & 0,9391 & 0,9687 & $-0,9 \%$ & $3,2 \%$ \\
\hline Barcelona & 1,0152 & 0,9761 & 0,9566 & 0,9587 & $-5,6 \%$ & $0,2 \%$ \\
\hline Navarra & 0,9653 & 0,8956 & 0,9249 & 0,9400 & $-2,6 \%$ & $1,6 \%$ \\
\hline Burgos & 0,9619 & 0,9320 & 0,9114 & 0,9378 & $-2,5 \%$ & $2,9 \%$ \\
\hline Alicante/Alacant & 0,8951 & 0,9045 & 0,9066 & 0,9355 & $4,5 \%$ & $3,2 \%$ \\
\hline Bizkaia & 0,8599 & 0,8855 & 0,8739 & 0,9309 & $8,3 \%$ & $6,5 \%$ \\
\hline Palencia & 0,9556 & 0,9445 & 0,9117 & 0,9240 & $-3,3 \%$ & $1,4 \%$ \\
\hline Murcia & 0,9026 & 0,8536 & 0,8908 & 0,9144 & $1,3 \%$ & $2,6 \%$ \\
\hline Ourense & 0,9961 & 0,9161 & 0,8804 & 0,9101 & $-8,6 \%$ & $3,4 \%$ \\
\hline Segovia & 0,9116 & 0,9252 & 0,8816 & 0,9089 & $-0,3 \%$ & $3,1 \%$ \\
\hline León & 0,9501 & 0,9327 & 0,9090 & 0,9050 & $-4,7 \%$ & $-0,4 \%$ \\
\hline Valencia/València & 0,8712 & 0,8733 & 0,8765 & 0,9047 & $3,8 \%$ & $3,2 \%$ \\
\hline Rioja, La & 0,9304 & 0,8853 & 0,8945 & 0,9024 & $-3,0 \%$ & $0,9 \%$ \\
\hline Albacete & 0,8809 & 0,9309 & 0,8650 & 0,9019 & $2,4 \%$ & $4,3 \%$ \\
\hline Soria & 0,9396 & 0,9529 & 0,9398 & 0,9001 & $-4,2 \%$ & $-4,2 \%$ \\
\hline Araba/Álava & 0,8809 & 0,8850 & 0,8832 & 0,8973 & $1,9 \%$ & $1,6 \%$ \\
\hline Ávila & 0,9427 & 0,9066 & 0,9047 & 0,8916 & $-5,4 \%$ & $-1,4 \%$ \\
\hline Zamora & 0,9620 & 0,9291 & 0,9224 & 0,8858 & $-7,9 \%$ & $-4,0 \%$ \\
\hline Salamanca & 0,9088 & 0,9248 & 0,8851 & 0,8808 & $-3,1 \%$ & $-0,5 \%$ \\
\hline Teruel & 0,9217 & 0,8995 & 0,8845 & 0,8737 & $-5,2 \%$ & $-1,2 \%$ \\
\hline Cuenca & 0,9344 & 0,8980 & 0,8707 & 0,8706 & $-6,8 \%$ & $0,0 \%$ \\
\hline Guadalajara & 0,8435 & 0,8929 & 0,8453 & 0,8682 & $2,9 \%$ & $2,7 \%$ \\
\hline Huesca & 0,8479 & 0,8196 & 0,8034 & 0,8614 & $1,6 \%$ & $7,2 \%$ \\
\hline Badajoz & 0,8779 & 0,8781 & 0,8642 & 0,8580 & $-2,3 \%$ & $-0,7 \%$ \\
\hline Zaragoza & 0,9186 & 0,8788 & 0,9005 & 0,8560 & $-6,8 \%$ & $-4,9 \%$ \\
\hline Valladolid & 0,8556 & 0,8632 & 0,8344 & 0,8521 & $-0,4 \%$ & $2,1 \%$ \\
\hline Toledo & 0,8816 & 0,8838 & 0,8533 & 0,8458 & $-4,1 \%$ & $-0,9 \%$ \\
\hline Cáceres & 0,8989 & 0,8673 & 0,8543 & 0,8456 & $-5,9 \%$ & $-1,0 \%$ \\
\hline Jaén & 0,8399 & 0,8730 & 0,8478 & 0,8432 & $0,4 \%$ & $-0,5 \%$ \\
\hline Córdoba & 0,8605 & 0,8380 & 0,8349 & 0,8345 & $-3,0 \%$ & $-0,1 \%$ \\
\hline Madrid & 0,8340 & 0,8498 & 0,8183 & 0,8339 & $0,0 \%$ & $1,9 \%$ \\
\hline Ciudad Real & 0,8921 & 0,8723 & 0,8538 & 0,8313 & $-6,8 \%$ & $-2,6 \%$ \\
\hline Sevilla & 0,8614 & 0,8186 & 0,8102 & 0,8310 & $-3,5 \%$ & $2,6 \%$ \\
\hline Palmas, Las & 0,8252 & 0,8284 & 0,8441 & 0,8300 & $0,6 \%$ & $-1,7 \%$ \\
\hline Granada & 0,8181 & 0,8004 & 0,8247 & 0,8263 & $1,0 \%$ & $0,2 \%$ \\
\hline Santa Cruz & 0,8176 & 0,8203 & 0,8013 & 0,8035 & $-1,7 \%$ & $0,3 \%$ \\
\hline Lleida & 0,8227 & 0,7886 & 0,7983 & 0,7790 & $-5,3 \%$ & $-2,4 \%$ \\
\hline TOTAL NACIONAL & 0,9761 & 0,9723 & 0,9613 & 0,9796 & $0,4 \%$ & $1,9 \%$ \\
\hline
\end{tabular}


más concentradas, por ejemplo, coinciden en ambos listados. Por ejemplo, se confirma la evolución desfavorable de las Balears y el litoral andaluz, territorios ya caracterizados por una concentración comparativamente elevada. Asimismo, se reafirma la buena evolución, al menos en el período 1999-2012, de las provincias catalanas costeras, junto a Castellón y de las gallegas y cantábricas, todas ellas típicamente de elevada concentración.

\section{EXAMEN DE ALGUNOS FACTORES CORRELACIONADOS}

En este apartado se pretende abordar de una manera muy primigenia los posibles factores que estarían asociados a la concentración de la actividad turística provincial, y a sus diferencias. Para ello se han evaluado, utilizando el coeficiente de correlación parcial, la conexión estadística de factores como el volumen de demanda, el tipo de producto marcado por la localización geográfica, el mercado, o el clima.

En particular, se evalúa si un mayor volumen de demanda puede asociarse a la incidencia de la concentración temporal, dadas las restricciones masivas de los turistas en origen asociadas a las vacaciones laborales y escolares. La variable de demanda global coincide con el montante anual provincial de las pernoctaciones hoteleras. Asimismo, se han evaluado la incidencia de variables ligadas al producto, como la localización geográfica (provincia costera o interior) y una más particular asociada a la especialización en sol y playa de la zona mediterránea y sur. Asimismo, se contrasta si el tipo de mercado está significativamente asociado a la concentración de la actividad y, en particular, el peso del mercado nacional. En este sentido, esperaríamos una correlación negativa dado, por ejemplo, la mayor incidencia en este mercado de los viajes de fin de semana. Finalmente, se ha incluido una seria de variables aproximativas de la región climática en la que las provincias se inserta y que irían desde la región Norte, Sur, Mediterránea o Canarias 5 . Todas las variables, excepto la de mercado, son dicotómicas. Los cálculos se reproducen en el Cuadro 6 para años seleccionados del período. Los resultados, asimismo, se ofrece para los cálculos de la concentración tanto para una base mensual como cuatrimestral. Los cálculos ilustran, por ejemplo, que típicamente la demanda global, ser provincia costera, estar especializada en sol y playa o formar parte de la región climática mediterránea son rasgos asociados a una mayor concentración temporal de la actividad turística. Por el contrario, ser provincia interior, de la zona climática Sur, ser provincia canaria o con importante peso del mercado nacional son rasgos que tienden a atenuar este desequilibrio.

5 En Duro, Farré y Domonkos (2013) se describe, por ejemplo, la relevancia y contenido de estas regiones. En todo caso, su composición seria la siguiente:

Región Climática Norte: Álava, Asturias, Vizcaya, Burgos, Cantabria, Coruña, Guipúzcoa, Huesca, Lleida, Lugo, Navarra, Ourense, Palencia, Pontevedra, Rioja, Soria, Valladolid, Zamora y Zaragoza

Región Climática Sur: Albacete, Ávila, Badajoz, Cáceres, Ciudad Real, Córdoba, Cuenca. Guadalajara, Jaén, Madrid, Salamanca Segovia, Sevilla, Teruel, Toledo.

Región Climática Mediterránea: Alicante, Almería, Baleares, Barcelona, Cádiz, Castellón, Girona, Granada, Huelva, Málaga, Murcia, Tarragona y Valencia. 


\section{Cuadro 6 \\ COEFICIENTES DE CORRELACIÓN PARCIAL ENTRE ÍNDICES DE CONCENTRACIÓN TEMPORAL (C.V.) Y FACTORES, AÑOS SELECCIONADOS PERÍODO}

\begin{tabular}{|lcccc|}
\hline & $\mathbf{1 9 9 9}$ & $\mathbf{2 0 0 4}$ & $\mathbf{2 0 0 8}$ & $\mathbf{2 0 1 2}$ \\
\hline Base Mensual & & & & \\
Concentración-Demanda & 0.16 & $0.31^{*}$ & $0.31^{*}$ & $0.25^{*}$ \\
Concentración-Costa & $0.45^{*}$ & $0.47^{*}$ & $0.49^{*}$ & $0.58^{*}$ \\
Concentración-SyP & 0.24 & $0.31^{*}$ & $0.42^{*}$ & $0.46^{*}$ \\
Concentración-Int\&Rur & $-0.28^{*}$ & $-0.36^{*}$ & $-0.47^{*}$ & $-0.51^{*}$ \\
Concentración Mercado & -0.08 & -0.18 & -0.26 & -0.26 \\
Concentración-Reg ClimNOR & 0,17 & 0,06 & 0,03 & 0,01 \\
Concentración- Reg ClimSUR & $-0,41^{*}$ & $-0,37^{*}$ & $-0,44^{*}$ & $-0,45^{*}$ \\
Concentración- Reg ClimMED & $0,38^{*}$ & $0,43^{*}$ & $0,55^{*}$ & $0,60^{*}$ \\
Concentración- CAN & $-0,30^{*}$ & $-0,24$ & $-0,24$ & $-0,26$ \\
\hline Base Cuatrimestral & & & & \\
Concentración-Demanda & 0.24 & $0.37^{*}$ & $0.38^{*}$ & $0.35^{*}$ \\
Concentración-Costa & $0.47^{*}$ & $0.48^{*}$ & $0.52^{*}$ & $0.60^{*}$ \\
Concentración-SyP & 0.26 & $0.30^{*}$ & $0.43^{*}$ & $0.45^{*}$ \\
Concentración-Int\&Rur & $-0.32^{*}$ & $-0.37^{*}$ & $-0.48^{*}$ & $-0.51^{*}$ \\
Concentración Mercado & -0.17 & -0.22 & $-0.32^{*}$ & $-0.33^{*}$ \\
Concentración-Reg ClimNOR & 0,10 & 0,01 & $-0,03$ & $-0,01$ \\
Concentración- Reg ClimSUR & $-0,37^{*}$ & $-0,32^{*}$ & $-0,40^{*}$ & $-0,43^{*}$ \\
Concentración- Reg ClimMED & $0,39^{*}$ & $0,42^{*}$ & $0,54^{*}$ & $0,57^{*}$ \\
Concentración- CAN & $-0,25$ & $-0,23$ & $-0,20$ & $-0,22$ \\
\hline
\end{tabular}

Nota: * significativo a un nivel del $5 \%$. El vector demanda se mide a través del total de pernoctaciones hoteleras anuales; la variable Costa es una variable dicotómica (dummy) que toma el valor 1 en el caso de provincias con litoral y 0 en el caso de provincias interiores; la variable SyP es una variable dicotómica (dummy) que toma valor 1 en el caso de provincias costeras con litoral en el mediterráneo y Sur de la península; Int\&Tur es una variable dicotómica que toma valor 1 en el caso de provincias interiores y del norte; Mercado es una variable asociada al peso del mercado nacional sobre el total anual; RegClimNOR (provincias del norte de España), SUR (provincia centro y sur sin costa mediterráneo) y $M E D$ (provincias con litoral costero mediterráneo) son variables dicotómicas referidas a la región climática en la que se incluye cada provincia; y $C A N$ es una variable dicotómica que toma valor 1 en el caso de que sea una provincia canaria.

Fuente: Elaboración propia a partir de datos de la Encuesta de Ocupación Hotelera (EOH), INE.

En todo caso, los resultados anteriores no deben ser percibidos como algo automático. Así, éstos deben interpretarse en términos generales y no particulares. Por ejemplo, los efectos potencialmente desequilibradores del crecimiento de la demanda pueden atenuarse a partir de diferentes políticas o combinación de las mismas. Weaver y Opermann (2000) nos hablan de diferentes estrategias bien para aumentar la demanda en los meses de menor actividad o para reducirla en los de mayor demanda. En el primer caso, y que pueden ser las más políticamente atractivas, la literatura habla de tres palancas principales: en primer lugar, las medidas tendentes a modificar y diversificar el producto (eventos, festivales, etc); en segundo lugar, los instrumentos y política para captar mercados menos estacionales o compensatorios como seniors o de negocios, por ejemplo; y en tercer lugar, 
habría que reseñar las estrategias de precios, obviamente reduciéndolos ${ }^{6}$. Por otra parte, la reducción de la concentración puede necesitar la reducción de la actividad en los meses más saturados, enfatizando estrategias como las relacionadas con precios (crecimiento) o el establecimiento de capacidad de carga, por ejemplo, en áreas protegidas. En definitiva, no combatir la estacionalidad, dado el contexto descrito, puede amenazar la capacidad de crecimiento sostenible de los destinos, en su triple vertiente económica, social y ambiental.

\section{CONCLUSIONES FINALES}

En este trabajo se ha examinado el nivel de concentración temporal de la actividad turística en las provincias españolas a lo largo del período 1999-2012 y tomando como indicador de referencia las pernoctaciones hoteleras. Para ello en primer lugar se incide en la relevancia fundamental de las medidas de concentración a analizar, habida cuenta de su sensibilidad respecto a los resultados. En este punto, el trabajo recomienda el uso de diferentes medidas satisfactorias al mismo tiempo. En el apartado empírico el análisis muestra diferentes puntos de interés:

En primer lugar, los niveles de concentración temporal son muy variados a lo largo de la geografía provincial. En todo caso, se destacan, como las provincias unánimemente más concentradas las Baleares y las costeras catalanas, exceptuando el caso de la provincia de Barcelona. En el polo contrario, se destaca la bajísima estacionalidad en las provincias canarias o en Madrid.

En segundo lugar, y respecto a la evolución, el ascenso global de la concentración en el período, y que ha sido, por ejemplo, del 5.7\% según el coeficiente de variación, se reparte de manera muy diversa a lo largo de territorio. Provincias de elevada concentración como Baleares y las costeras andaluzas (excepto Granada, presumiblemente por su potente producto patrimonial) han registrado incluso aumentos superiores a la media nacional en el período. En contraste, otras provincias de concentración elevada como Tarragona o Girona, gallegas y cantábricas han revelado un patrón positivo en este sentido.

En tercer lugar, la observación con detenimiento de los resultados en función de las medidas de concentración utilizadas reflejan algunas discrepancias, aunque en su globalidad no son especialmente significativas.

En cuarto lugar, la realización de los cómputos anteriores sobre una base cuatrimestral, en lugar de mensual, arroja resultados globalmente semblantes.

Finalmente, se ha examinado, como punto de partida para modelos de explicación superiores, el grado de correlación entre una serie de factores potencialmente explicativos y las variaciones provinciales en la concentración temporal de la demanda turística. En particular, se han probado variables relacionadas con la magnitud de la demanda, el tipo cualitativo de producto, el área climática y el peso del mercado nacional. En síntesis, los resultados iniciales ilustran, por ejemplo, que típicamente el montante de demanda global, ser provincia costera, estar especializada en sol y playa o formar parte de la región climática mediterránea son rasgos asociados a una mayor concentración temporal de la

6 Roselló et al. (2004) por ejemplo descubrieron el efecto de las políticas de precios para reducir la estacionalidad en las Islas Baleares en los casos de los mercados británico (sobretodo) y alemán, de hecho, los dos mercados más importantes. 
actividad turística. Por el contrario, ser provincia interior, pertenecer a la zona climática Sur, ser provincia canaria o con importante peso del mercado nacional son rasgos que tienden a atenuar este desequilibrio.

\section{BIBLIOGRAFÍA}

ALLOCK, J. (1994): «Seasonality», en Tourism Marketing and Management Handbook, Witt, S.F. y Motuinho, L. (Eds.), Prentice Hall, NY, pp. 86-92.

BARON, R.V. (1975): Seasonality in Tourism-A Guide to the Analysis of Seasonality and Trends for Policy Making, Technical Series, 2, Economist Intelligence Unit, London

BARRO, R. y SALA-I-MARTIN, X. (1992): «Convergence», Journal of Political Economy, 100 (2), 223-251.

BUTLER, R.W., (2001), «Seasonality in tourism: Issues and implications», en BAUM, T. y LUNDTORP, S. (Eds.): Seasonality in tourism, pp. 5-22, Pergamon-Elsevier, Oxford.

CAPO, J., RIERA, A. y ROSELLÓ, J. (2007): «Accomodation determinants of seasonal patterns», Annals of Tourism Research, 34, pp. 422-436.

CHAKRAVORTY, S.R. (1990): Ethical Social Index Numbers, Springer Verlag, Berlin.

COWELL, F. (1995): Measuring Inequality, Second edition. New York: Prentice Hall.

CUCCIA, T. y RIZZO, I. (2011): «Tourism seasonality in cultural destinations. Empirical evidence from Sicily», Tourism Management, 32, pp. 589-595.

DURO, J.A. (2008): «La concentración temporal de la demanda turística en España y sus regiones: un análisis empírico a partir de índices de desigualdad», Revista de Análisis Turístico, 6, pp. 36-48.

DURO, J.A., FARRÉ, F.X. y DOMONKOS, P. (2013): «Climate Potential in Spanish regions: analysis and its relationship with tourism flows», mimeo.

FERNANDEZ-MORALES, A., (2003): «Decomposing seasonal concentration», Annals of Tourism Research, 30 (4), pp. 942-956.

FERNANDEZ-MORALES, A. y CRUZ-MAYORGA, M.C. (2008): «Seasonal concentration of the hotel demand in Costa del Sol: A decomposition by nationalities», Tourism Management, 29, pp. 940-949.

KOENIG-LEWIS, N. y BISCHOFF, E. (2005): «Seasonality research: the state of the art», International Journal of Tourism Research, 7, pp. 201-219.

GINI, C., (1912): Variabilità e mutabilità, C.Cuppini, Bologna.

LOPEZ, J. y LÓPEZ, L. (2006): «La concentración estacional en las regiones españolas desde una perspectiva de la oferta turística», Revista de Estudios Regionales, 77, pp. 77-104.

LUNDTORP, S. (2001): «Measuring tourism seasonality» en: BAUM, T. y LUNDTORP, S. (Eds.), Seasonality in tourism, pp. 23-50, Pergamon-Elsevier, Oxford.

MARTIN, J.M., JIMÉNEZ, J. y MOLINA, V., (2014): «Impacts of seasonality on environmental sustainability in the tourism sector based on destination type: an application to Spain's Andalusia region», Tourism Economics, 20 (1), pp. 123-142.

ROSELLÓ, J.A., RIERA, A. y SANSO, A. (2004): «The economic determinants of Seasonal patterns», Annals of Tourism Research, 31, pp. 697-711.

WANHILL, S. (1980): «Tackling seasonality: A technical note», International Journal of Tourism Management, 1(4), pp. 84-98. 